\title{
The Moderating Effect of Sleep Disturbance on the Association of Stress with Impulsivity and Depressed Mood
}

\author{
Inkyung Park ${ }^{1 *}$, Seong Min $\mathrm{Oh}^{2 *}$, Kyung Hwa Lee ${ }^{3}$, Soohyun $\mathrm{Kim}^{4}$, Jeong Eun Jeon ${ }^{5}$, \\ Ha Young Lee ${ }^{5}$, Sehyun Jeon ${ }^{6}$, Seog Ju Kim ${ }^{6}$, and Yu Jin Lee ${ }^{5 凶}$ \\ 1Department of Psychological and Brain Sciences, University of lowa, lowa City, lowa, USA \\ ${ }^{2}$ Department of Psychiatry, Dongguk University Ilsan Hospital, Goyang, Republic of Korea \\ ${ }^{3}$ Division of Child and Adolescent Psychiatry, Department of Psychiatry, Seoul National University Hospital, Seoul, Republic of Korea \\ ${ }^{4}$ Department of Neurology, Gangneung Asan Hospital, Gangneung, Republic of Korea \\ ${ }^{5}$ Department of Psychiatry and Center for Sleep and Chronobiology, Seoul National University Hospital, Seoul, Republic of Korea \\ ${ }^{6}$ Department of Psychiatry, Sungkyunkwan University School of Medicine, Samsung Medical Center, Seoul, Republic of Korea
}

Objective This study was performed to investigate the associations of life event stress with impulsivity, anxiety, and depressed mood as a function of the presence of a sleep disturbance.

Methods In total, 214 participants (age 38.96 10.53 years; 111 females) completed self-report questionnaires, including the Life Experience Survey (LES), Pittsburgh Sleep Quality Index (PSQI), Barratt's Impulsivity Scale (BIS), Beck Anxiety Inventory (BAI), and Beck Depression Inventory (BDI). The presence of a sleep disturbance was defined as a PSQI score $>5$.

Results In total, 127 participants presented with a sleep disturbance (age $39.33 \pm 10.92$ years; 64 females), whereas the remaining 87 did not (age 38.43 \pm 9.97 years; 47 females). Negative LES scores were significantly correlated with $B I S(r=0.22, p=0.001), B A I(r=0.46, p<$ $0.001)$, and BDI $(r=0.51, p<0.001)$ scores, and PSQI scores were significantly correlated with BAI $(r=0.49, p<0.001)$ and $B D I(r=0.60$, $p<$ $0.001)$ scores. Moderation analysis revealed statistically significant interactions between negative LES scores and the presence of a sleep disturbance on BIS ( $\mathrm{p}=0.044)$ and BDI $(\mathrm{p}=0.014)$ but not on BAI $(\mathrm{p}=0.194)$ scores.

Conclusion The findings of the present study suggest that life event stress has varying degrees of influence on mental health, especially impulsivity and depressed mood, depending on the presence or absence of a sleep disturbance. Psychiatry Investig 2020;17(3):243-248

Key Words Sleep-wake disorders, Psychological stress, Mental health.

\section{INTRODUCTION}

Modern life is associated with various stressful events, which are known to have an effect on both physiological and psychological health. ${ }^{1,2}$ Specifically, the high stress levels caused by ev-

\footnotetext{
Received: July 16, 2019 Revised: October 17, 2019

Accepted: November 18, 2019

$\triangle$ Correspondence: Seog Ju Kim, MD, PhD

Department of Psychiatry, Sungkyunkwan University School of Medicine, Samsung Medical Center, 81 Irwon-ro, Gangnam-gu, Seoul 06351, Republic of Korea

Tel: +82-2-3410-3583, Fax: +82-2-3410-0050, E-mail: ksj7126@skku.edu

$\triangle$ Correspondence: Yu Jin Lee, MD, PhD

Department of Psychiatry and Center for Sleep and Chronobiology, Seoul National University College of Medicine, 103 Daehak-ro, Jongno-gu, Seoul 03080, Republic of Korea

Tel: +82-2-2072-2456, Fax: +82-2-744-7241, E-mail: ewpsyche@snu.ac.kr

*These authors contributed equally to this work.

(a) This is an Open Access article distributed under the terms of the Creative Commons Attribution Non-Commercial License (https://creativecommons.org/licenses/by$\mathrm{nc} / 4.0$ ) which permits unrestricted non-commercial use, distribution, and reproduction in any medium, provided the original work is properly cited.
}

eryday life events (i.e., life event stress) are clearly associated with symptoms of mood disorders, including depression and anxiety. ${ }^{3-6}$ Other studies have reported a positive correlation between life event stress and impulsivity. ${ }^{7.8}$

The harmful effect of sleep disturbance on mental health has also been well documented. Individuals with sleep disturbance have been reported to show increased depressive mood and anxiety. ${ }^{9-12}$ Baglioni et al. ${ }^{10}$ reported that individuals with sleep disturbance have twice the risk of developing depression compared to those without sleep disturbance, and Spoormaker and van den Bout. ${ }^{12}$ reported that anxiety is one of the most common comorbidities in patients with insomnia. Other studies have shown that sleep disturbance is also significantly correlated with impulsivity. ${ }^{13,14}$ For example, a study using a sleep loss paradigm indicated that sleep-deprived individuals show increased impulsivity in response to negative stimuli. ${ }^{14}$

Both life event stress and sleep disturbance have well-established relationships with impulsivity, anxiety, and depressed 
mood. However, it is important to note that stress and sleep disturbance are closely connected phenomena, and stress plays a role as a significant predictor of sleep disturbance. ${ }^{15,16}$ Drake et al. suggested that dispositional vulnerability to sleep disturbance in the presence of stressors may be a predictive factor for mental disorders, such as depression. ${ }^{17-19}$ Therefore, it may be beneficial to investigate the effects of the interaction of life event stress and sleep disturbance on mental health.

To our knowledge, there have been no previous studies regarding the effects of the interaction of life event stress and sleep disturbance on mental health. In the present study, we examined how the associations of life event stress with impulsivity, anxiety, and depressed mood change in the presence of sleep disturbance. Both life event stress and sleep disturbance were postulated to be associated with impulsivity, anxiety, and depressed mood, which is consistent with the findings of previous studies. We also hypothesized that the presence of sleep disturbance may mediate the associations of life event stress with impulsivity, anxiety, and depressed mood.

\section{METHODS}

\section{Participants and procedure}

Participants in the present study were recruited via ads placed on message boards in the Center for Sleep and Chronobiology at Seoul National University Hospital. Individuals with psychiatric, medical, and sleep disorders were excluded, and 227 healthy adults were enrolled. Participants were asked to respond to questionnaires, including the Life Experience Survey (LES), ${ }^{20,21}$ Pittsburgh Sleep Quality Index (PSQI), ${ }^{22,23}$ Barratt's Impulsivity Scale (BIS), ${ }^{24,25}$ Beck Anxiety Inventory (BAI), ${ }^{26,27}$ and Beck Depression Inventory (BDI). ${ }^{28,29}$ The study protocol was approved by the Institutional Review Board of Seoul National University Hospital (IRB No. 1608-160-788). All participants provided written informed consent after receiving a full description of the study.

\section{Assessments}

The LES is a self-report measure of experience with $60 \mathrm{ma}-$ jor life events that may have occurred in the recent past. Of the 60 original items, we excluded 10 designed for students, leaving 50 items on the final measure. Each event that the respondent has encountered within past 6 months is rated from -3 (extremely negative) to +3 (extremely positive). The present study used the absolute value of the sum of the negative event ratings as an index of participants' life event-related stress levels; higher scores indicated higher levels of stress.

The PSQI is a questionnaire measuring the overall quality of sleep within the past 4 weeks. It consists of 19 self-rated items divided into seven component scores ranging from 0 to 3 : sub- jective sleep quality, sleep latency, sleep duration, sleep efficiency, sleep disturbances, use of sleep medications, and daytime dysfunction. The sum of the seven components, the global PSQI score, can range between 0 and 21 , with global scores $>5$ indicating a poor quality of sleep.

The BIS is a 23-item self-report measure that assesses impulsive tendencies. The sum of all the items reflects overall impulsivity, with higher BIS scores indicating higher levels of impulsivity.

The BAI is a tool used to measure the common symptoms of anxiety. The scale consists of 21 items, and higher BAI scores indicate more anxiety.

The BDI is a 21-item self-report measure that assesses the severity of depressive symptoms. Higher BDI scores indicate more severe depressive symptoms.

\section{Statistical analysis}

Descriptive statistics on age, gender, and clinical variables were calculated. Self-report questionnaire scores more than three standard deviations above or below the mean were regarded as outliers and were excluded from the analysis. Participants were also divided into two groups, poor sleepers (PS) and good sleepers (GS), according to whether their PSQI scores were $>5$. The $t$ test was used to compare the differences between the groups in negative LES, BIS, BAI, and BDI scores. Correlation analyses were also performed to examine the associations of negative LES and PSQI scores with BIS, BAI, and BDI scores. Partial correlation coefficients adjusted for age and gender were calculated. Finally, three separate moderation analyses were performed to investigate whether the effects of negative LES scores on BIS, BAI, and BDI scores were moderated by the presence of a sleep disturbance (i.e., PS group vs. GS group). Moderation analysis is used to determine the conditions under which the strength of an association between two variables varies. Instead of using fixed regression coefficients for covariates, in the moderation analysis, we treated the regression coefficient as a function of the independent variable to analyze the moderating effect of the covariate. The negative LES, BIS, BAI, and BDI scores were mean-centered in the current moderation analyses to avoid multicollinearity given the high intercorrelations. IBM SPSS 21 (IBM Corp., Armonk, NY, USA) was used for all statistical calculations, and PROCESS macro for SPSS was used to carry out moderation analyses. ${ }^{30}$

\section{RESULTS}

\section{Participants characteristics}

Thirteen of the total of 227 participants were excluded due to incomplete questionnaires, and 214 (mean age \pm SD, 38.96 \pm 10.53 years; 111 females) were included in the final analysis. 
Table 1. Baseline characteristics of the 214 participants analyzed in the study

\begin{tabular}{lcccc}
\hline \multicolumn{1}{c}{ Variable } & Total $(\mathrm{N}=214)$ & Poor sleeper $(\mathrm{N}=127)$ & Good sleeper $(\mathrm{N}=87)$ & $\mathrm{p}$ \\
\hline Age (years) & $38.96 \pm 10.53$ & $39.33 \pm 10.92$ & $38.43 \pm 9.97$ & 0.538 \\
Females (\%) & $111(51.9)$ & $64(50.4)$ & $47(54.0)$ & 0.602 \\
Negative LES & $4.39 \pm 4.87$ & $5.68 \pm 5.18$ & $2.52 \pm 3.67$ & $<0.001$ \\
PSQI & $6.94 \pm 3.52$ & $9.06 \pm 3.01$ & $3.85 \pm 1.06$ & $<0.001$ \\
BIS & $57.52 \pm 9.85$ & $57.92 \pm 10.67$ & $56.93 \pm 8.54$ & 0.454 \\
BAI & $8.79 \pm 8.39$ & $11.29 \pm 8.62$ & $5.15 \pm 6.52$ & $<0.001$ \\
BDI & $8.55 \pm 8.09$ & $11.48 \pm 8.78$ & $4.27 \pm 4.25$ & $<0.001$ \\
\hline
\end{tabular}

Values are presented as means \pm SD or N (\%). Poor Sleepers are defined as PSQI score $>5$, Good Sleepers as PSQI $\leq 5$. Negative LES: negative Life Experience Survey, PSQI: Pittsburgh Sleep Quality Index, BIS: Barratt's Impulsivity Scale, BAI: Beck Anxiety Inventory, BDI: Beck Depression Inventory

Baseline characteristics are summarized in Table 1. Based on a PSQI cutoff score of 5, the PS group consisted of 127 participants (mean age $\pm S D, 39.33 \pm 10.92$ years; 64 females), and the

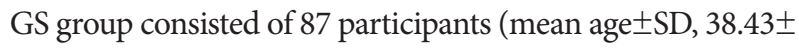
9.97 years; 47 females). There were no significant differences in age, gender, or BIS scores between the PS and GS groups. The PS group had significantly higher negative LES (mean \pm SD, $5.68 \pm 5.18$ vs. $4.39 \pm 4.87$, respectively; $\mathrm{p}<0.001$ ), PSQI (mean \pm $\mathrm{SD}, 9.06 \pm 3.01$ vs. $6.94 \pm 3.52$, respectively; $\mathrm{p}<0.001$ ), BAI (mean $\pm \mathrm{SD}, 11.29 \pm 8.62$ vs. $8.79 \pm 8.39$, respectively; $\mathrm{p}<0.001$ ), and BDI (mean \pm SD, $11.48 \pm 8.78$ vs. $8.55 \pm 8.09$, respectively; $\mathrm{p}<0.001$ ) scores compared to the GS group.

\section{Correlational of negative LES and PSQI with BIS, BAI, and BDI}

Table 2 shows the correlations between the age- and genderadjusted negative LES and PSQI scores with the BIS, BAI, and BDI scores in the total sample. Negative LES scores were significantly positively correlated with BIS $(r=0.22, p=0.001)$, BAI $(\mathrm{r}=0.46, \mathrm{p}<0.001)$, and BDI $(\mathrm{r}=0.51, \mathrm{p}<0.001)$ scores. PSQI scores were significantly positively correlated with BAI $(r=$ $0.49, \mathrm{p}<0.001)$ and BDI $(\mathrm{r}=0.60, \mathrm{p}<0.001)$ scores but not with BIS scores $(r=0.11, p=0.120)$.

\section{Moderating effect of the presence of sleep disturbance on the relationship of negative LES with BIS, BAI, and BDI}

We examined the role of sleep disturbance (i.e., PS vs. GS) as a moderator of the relationships between negative LES scores and BIS, BAI, and BDI scores. The results of the moderation analysis are summarized in Table 3 . First, the presence of a sleep disturbance had a significant moderating effect on the relationship between negative LES and BIS scores ( $\mathrm{p}=0.044$ ). In the PS group, a high negative LES score was significantly associated with a high BIS score, but this was not the case in the GS group (Figure 1A; PS, B=0.60, p=0.001; GS, B=-0.06, p= 0.800 ). Second, the presence of a sleep disturbance did not have
Table 2. Age- and gender-adjusted relationships of negative LES and PSQI scores with mental health variables

\begin{tabular}{|c|c|c|c|c|c|c|}
\hline \multirow{2}{*}{ Predictor } & \multicolumn{2}{|c|}{ BIS } & \multicolumn{2}{|c|}{ BAI } & \multicolumn{2}{|c|}{ BDI } \\
\hline & $\mathrm{r}$ & $\mathrm{p}$ & $\mathrm{r}$ & $\mathrm{p}$ & $\mathrm{r}$ & $\mathrm{p}$ \\
\hline Negative LES & 0.22 & 0.001 & 0.46 & $<0.001$ & 0.51 & $<0.001$ \\
\hline PSQI & 0.11 & 0.120 & 0.49 & $<0.001$ & 0.60 & $<0.001$ \\
\hline
\end{tabular}

Negative LES: negative Life Experience Survey, PSQI: Pittsburgh Sleep Quality Index, BIS: Barratt's Impulsivity Scale, BAI: Beck Anxiety Inventory, BDI: Beck Depression Inventory

a significant moderating effect on the relationship between negative LES and BAI scores $(\mathrm{p}=0.194)$. Finally, the effect of the interaction between negative LES scores and the presence of a sleep disturbance on BDI scores was statistically significant $(\mathrm{p}=0.014)$. Although negative LES scores showed a significant correlation with BDI scores in both groups, the correlation was stronger in the PS group than in the GS group (Figure $1 \mathrm{~B} ; \mathrm{PS}, \mathrm{B}=0.85, \mathrm{p}<0.001$; $\mathrm{GS}, \mathrm{B}=0.30, \mathrm{p}=0.016$ ).

\section{DISCUSSION}

The present study found positive correlations between life event stress and impulsivity, anxiety, and depressed mood and positive correlations between sleep disturbance and anxiety and depressed mood. We also observed that sleep disturbance mediated the associations of life event stress with impulsivity and depressed mood. Life event stress was associated with increased impulsivity only in participants with a sleep disturbance. Moreover, life event stress was more strongly correlated with depressed mood in participants with a sleep disturbance than in those without a sleep disturbance. To our knowledge, this is the first study to investigate the moderating effects of sleep disturbances on associations between life event stress and mental health.

Our findings support those of previous studies showing that life event stress and sleep disturbances are associated with anxiety and depressed mood. Tennant's meta-analytic studies found 
Table 3. Moderation analysis of the interaction between the negative LES score and the presence of sleep disturbance (Poor Sleeper vs. Good Sleeper)

\begin{tabular}{|c|c|c|c|c|c|c|c|c|c|}
\hline \multirow{2}{*}{ Predictor } & \multicolumn{3}{|c|}{ BIS } & \multicolumn{3}{|c|}{ BAI } & \multicolumn{3}{|c|}{ BDI } \\
\hline & B & $\mathrm{p}$ & $95 \% \mathrm{CI}$ & B & $\mathrm{p}$ & $95 \% \mathrm{CI}$ & B & $\mathrm{p}$ & $95 \% \mathrm{CI}$ \\
\hline Negative LES (X) & 0.33 & 0.031 & $0.03,0.63$ & 0.63 & $<0.001$ & $0.41,0.85$ & 0.62 & $<0.001$ & $0.42,0.82$ \\
\hline Sleep disturbance (W) & -0.34 & 0.815 & $-3.21,2.53$ & -4.33 & 0.001 & $-6.48,-2.19$ & -5.57 & $<0.001$ & $-7.50,-3.64$ \\
\hline $\mathrm{X} \times \mathrm{W}$ & -0.66 & 0.044 & $-1.31,-0.02$ & -0.32 & 0.194 & $-0.80,0.16$ & -0.55 & 0.014 & $-0.98,-0.11$ \\
\hline
\end{tabular}

Poor Sleepers are defined as Pittsburgh Sleep Quality Index score >5, Good Sleepers as $\leq 5$. Negative LES: negative Life Experience Survey, BIS: Barratt's Impulsivity Scale, BAI: Beck Anxiety Inventory, BDI: Beck Depression Inventory
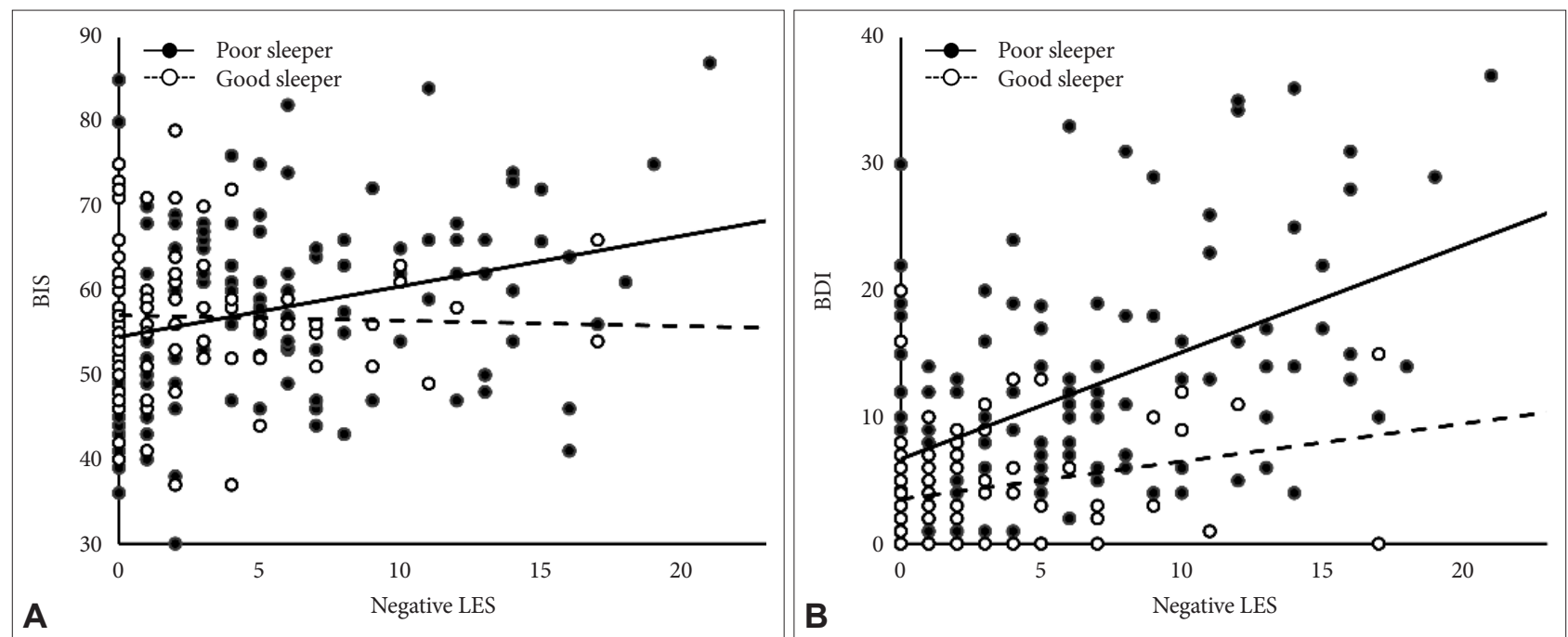

Figure 1. Scatter plot of negative LES score vs. BIS and BDI scores. Poor Sleepers are defined as PSQI scores $>5$, Good Sleepers as $P S Q I \leq 5$. (A) Life event stress, measured by sum of negative life event ratings in Life Experience Survey, was significantly correlated with increase in impulsivity, measured by Barratt's Impulsivity Scale, in poor sleeper group $(B=0.60, p=0.001)$, but it was not in good sleeper group $(B=-0.06, p=0.800)$. $(B)$ Life event stress was more highly correlated with increased depressed mood, measured by Beck Depression Inventory, in poor sleeper group $(B=0.85, p<0.001)$ than good sleeper group $(B=0.30, p=0.016)$. Negative LES: negative Life Experience Survey, BIS: Barratt's Impulsivity Scale, BAI: Beck Anxiety Inventory, BDI: Beck Depression Inventory.

that life event stressors were associated with increased risk of depression, ${ }^{3}$ and Williams et al. ${ }^{6}$ found a relationship between life event stress and anxiety. Spoormaker and Boult reported a strong association between sleep disturbances and depression/anxiety complaints. ${ }^{12}$ Our findings also suggest that life event stress and sleep disturbances play critical roles in stressinduced impulsivity, consistent with Anderson and Platten's ${ }^{14}$ report that sleep deprivation enhanced impulsive reactions to negative stimuli. A potential neural explanation is that decreased prefrontal activity and prefrontal-limbic connectivity in those with sleep disturbances ${ }^{31,32}$ may result in limbic system hyperactivation, which is associated with increased impulsiveness. ${ }^{33,34}$

The association between life event stress and depressed mood was more prominent in participants with a sleep disturbance than in those without a sleep disturbance. Thus, having a sleep disturbance may promote the development of a depressed mood in the context of life event stress. Although the mechanism by which a sleep disturbance facilitated the development of a depressed mood is not clear, a number of hypotheses have been proposed. One is a learned helplessness model in which those who had insomnia for a long period with little improvement may not even try to escape from it. This is one of the major psychological theories used to explain the development of depression. ${ }^{35}$ Hypercortisolism, observed in some of patients with insomnia, may also contribute to the development of depressed mood..$^{36}$ Those with a sleep disturbance, who are at increased risk of depression may become more depressed after life event stress in comparison with those who are not depressed.

Unlike impulsivity or depressed mood, we found no moderating effect of sleep disturbance on the association of anxiety with life event stress. Although the association between anxiety and sleep disturbance is widely acknowledged, ${ }^{9,12}$ there are controversies regarding the mechanism underlying this association. One prospective study investigated the directions of the relationship between anxiety and insomnia over a 1-year period, and the results indicated that, although anxiety has a bidirectional relationship with insomnia, it has more power as a predictor of future insomnia than vice versa. ${ }^{37}$ The role of anxiety as a cause of sleep disturbances may explain why such disturbances have little influence on stress-induced anxiety. 
This study had several limitations. First, it was difficult to confirm the causal relationships among recent life event stress, the presence of a sleep disturbance, and the mental health status of participants in this cross-sectional study. Additional research, including a longitudinal follow up to confirm the effects of the interaction of sleep disturbances and life event stress on mental health, is needed. Second, although we enrolled only healthy adults in this study, it is possible that undiagnosed comorbid factors, such as posttraumatic stress disorder, or sleep disorders, including obstructive sleep apnea, may have influenced sleep independent of the effects of impulsivity, anxiety, and depressed mood. Third, we measured self-reported sleep quality with a self-rating scale but did not objectively evaluate sleep characteristics with polysomnography. Finally, we surveyed negative life experiences over the most recent 6 months; thus, the effects of serious events that may have occurred earlier were not considered in this analysis.

The results of the present study suggest that life event stress has varying degrees of influence on mental health depending on the presence of a sleep disturbance. This possible association provided insight not only into the mechanism by which life event stress and sleep disturbance affect metal health but also into the search for therapeutic options for those who experience psychiatric problems after stressful events. Prospective studies will be required to determine whether improving sleep quality by medication or by cognitive-behavioral intervention is beneficial for reducing the mental health problems associated with life event stress.

\section{Acknowledgments}

This research was supported by the Basic Science Research Program through the National Research Foundation of Korea (Grant no. NRF2018R1D1A1B07049704 to Dr Yu Jin Lee), and by the Brain Research Program through the National Research Foundation of Korea funded by the Ministry of Science, ICT \& Future Planning (Grant no. 2016M3C7A1904336 to Dr Seog Ju Kim)

\section{Conflicts of Interest}

The authors have no potential conflicts of interest to disclose.

\section{Author Contributions}

Conceptualization: Yu Jin Lee, Seog Ju Kim. Data curation: Inkyung Park, Soohyun Kim, Jeong Eun Jeon, Ha Young Lee. Formal analysis: Inkyung Park, Seong Min Oh, Kyung Hwa Lee. Funding acquisition: Yu Jin Lee, Seog Ju Kim. Investigation: Inkyung Park, Soohyun Kim, Seong Min Oh. Methodology: Inkyung Park, Seong Min Oh. Project administration: Yu Jin Lee, Seog Ju Kim. Spervision: Yu Jin Lee, Seog Ju Kim. Visualization: Seong Min Oh. Writing-original draft: Inkyung Park, Seong Min Oh. Writing-review \& editing: Seong Min Oh.

\section{ORCID iDs}

Inkyung Park

Seong Min Oh

Kyung Hwa Lee

Soohyun Kim

Jeong Eun Jeon https://orcid.org/0000-0002-1681-6288

https://orcid.org/0000-0001-8895-8990

https://orcid.org/0000-0002-6168-5432

https://orcid.org/0000-0003-0424-8575

https://orcid.org/0000-0002-1723-0123
Ha Young Lee

Sehyun Jeon

Seog Ju Kim

Yu Jin Lee

https://orcid.org/0000-0002-7769-400X

https://orcid.org/0000-0002-6594-6556

https://orcid.org/0000-0003-2467-5451

https://orcid.org/0000-0001-5195-2579

\section{REFERENCES}

1. Andrews G, Tennant C. Life event stress and psychiatric illness. Psychol Med 1978;8:545-549.

2. Salleh MR. Life event, stress and illness. Malays J Med Sci 2008;15:9-18.

3. Tennant C. Life events, stress and depression: a review of recent findings. Aust N Z J Psychiatry 2002;36:173-182.

4. Kendler KS, Karkowski LM, Prescott CA. Causal relationship between stressful life events and the onset of major depression. Am J Psychiatry 1999;156:837-841.

5. Kessler RC. The effects of stressful life events on depression. Ann Rev Psychol 1997;48:191-214.

6. Williams JM, Tonymon P, Andersen MB. Effects of life-event stress on anxiety and peripheral narrowing. Behav Med 1990;16:174-181.

7. Lightsey Jr OR, Hulsey CD. Impulsivity, coping, stress, and problem gambling among university students. J Counsel Psychol 2002;49:202211.

8. Moustafa AA, Tindle R, Frydecka D, Misiak B. Impulsivity and its relationship with anxiety, depression and stress. Compr Psychiatry 2017; 74:173-179.

9. Nyer M, Farabaugh A, Fehling K, Soskin D, Holt D, Papakostas GI, et al. Relationship between sleep disturbance and depression, anxiety, and functioning in college students. Depress Anxiety 2013;30:873-880.

10. Baglioni C, Battagliese G, Feige B, Spiegelhalder K, Nissen C, Voderholzer $\mathrm{U}$, et al. Insomnia as a predictor of depression: a meta-analytic evaluation of longitudinal epidemiological studies. J Affect Disord 2011; 135:10-19.

11. Franzen PL, Buysse DJ. Sleep disturbances and depression: risk relationships for subsequent depression and therapeutic implications. Dialogues Clin Neurosci 2008;10:473-481.

12. Spoormaker VI, van den Bout J. Depression and anxiety complaints; relations with sleep disturbances. Eur Psychiatry 2005;20:243-245.

13. Van Veen MM, Karsten J, Lancel M. Poor sleep and its relation to impulsivity in patients with antisocial or borderline personality disorders. Behav Med 2017;43:218-226.

14. Anderson C, Platten CR. Sleep deprivation lowers inhibition and enhances impulsivity to negative stimuli. Behav Brain Res 2011;217:463466.

15. Healey ES, Kales A, Monroe LJ, Bixler EO, Chamberlin K, Soldatos CR. Onset of insomnia: role of life-stress events. Psychosom Med 1981; 43:439-451.

16. Hall M, Buysse DJ, Nowell PD, Nofzinger EA, Houck P, Reynolds III $\mathrm{CF}$, et al. Symptoms of stress and depression as correlates of sleep in primary insomnia. Psychosom Med 2000;62:227-230.

17. Drake C, Richardson G, Roehrs T, Scofield H, Roth T. Vulnerability to stress-related sleep disturbance and hyperarousal. Sleep 2004;27:285291.

18. Drake CL, Pillai V, Roth T. Stress and sleep reactivity: a prospective investigation of the stress-diathesis model of insomnia. Sleep 2014;37: 1295-1304.

19. Vargas I, Friedman NP, Drake CL. Vulnerability to stress-related sleep disturbance and insomnia: Investigating the link with comorbid depressive symptoms. Transl Issues Psychol Sci 2015;1:57-66.

20. Sarason IG, Johnson JH, Siegel JM. Assessing the impact of life changes: development of the Life Experiences Survey. J consult Clin Psychol 1978;46:932-946.

21. Lee YH. Relations between Attributional Style, Life Events, Event Attribution, Hopelessness and Depression. Unpublished doctoral dissertation. Seoul: Seoul National University; 1993.

22. Buysse DJ, Reynolds III CF, Monk TH, Berman SR, Kupfer DJ. The 
Pittsburgh Sleep Quality Index: a new instrument for psychiatric practice and research. Psychiatry Res 1989;28:193-213.

23. Sohn SI, Kim DH, Lee MY, Cho YW. The reliability and validity of the Korean version of the Pittsburgh Sleep Quality Index. Sleep Breath 2012;16:803-812.

24. Patton JH, Stanford MS, Barratt ES. Factor structure of the Barratt impulsiveness scale. J Clin Psychol 1995;51:768-774

25. Lee SR, Lee WH, Park JS, Kim SM, Kim JW, Shim JH. The study on Reliability and Validity of Korean Version of the Barratt Impulsiveness Scale-11-Revised in nonclinical adult subjects. J Korean Neuropsychiatr Assoc 2012;51:378-386.

26. Beck AT, Epstein N, Brown G, Steer RA. An inventory for measuring clinical anxiety: psychometric properties. J Consult Clin Psychol 1988; 56:893-897.

27. Yook SP, Kim ZS. A clinical study on the Korean version of Beck Anxiety Inventory: comparative study of patient and non-patient. Korean J Clin Psychol 1997;16:185-197.

28. Beck AT, Ward CH, Mendelson M, Mock J, Erbaugh J. An inventory for measuring depression. Arch Gen Psychiatry 1961;4:561-571.

29. Rhee M. A standardization study of Beck Depression Inventory I-Korean version (K-BDI): reliability and factor analysis. Korean J Psychopathol 1995;4:77-95.

30. Hayes AF. PROCESS: A Versatile Computational Tool for Observed
Variable Mediation, Moderation, and Conditional Process Modeling. 2012, [White Paper]. Available at: http://www.afhayes.com/public/process2012.pdf.

31. Chee MW, Choo WC. Functional imaging of working memory after $24 \mathrm{hr}$ of total sleep deprivation. J Neurosci 2004;24:4560-4567.

32. Yoo SS, Gujar N, Hu P, Jolesz FA, Walker MP. The human emotional brain without sleep-a prefrontal amygdala disconnect. Curr Biol 2007;17:R877-R888

33. New AS, Hazlett EA, Buchsbaum MS, Goodman M, Mitelman SA, Newmark R, et al. Amygdala-prefrontal disconnection in borderline personality disorder. Neuropsychopharmacology 2007;32:1629-1640.

34. Jentsch JD, Taylor JR. Impulsivity resulting from frontostriatal dysfunction in drug abuse: implications for the control of behavior by reward-related stimuli. Psychopharmacology 1999;146:373-390.

35. Seligman ME. Helplessness: On Depression, Development, and Death. A Series of Books in Psychology. New York, NY: WH Freeman/Times Books/Henry Holt \& Co; 1975.

36. Steiger A. Sleep and the hypothalamo-pituitary-adrenocortical system. Sleep Med Rev 2002;6:125-138.

37. Jansson-Fröjmark M, Lindblom K. A bidirectional relationship between anxiety and depression, and insomnia? A prospective study in the general population. J Psychosom Res 2008;64:443-449. 